\title{
Cryptocurrency reaction to FOMC Announcements: Evidence of Heterogeneity Based on Blockchain Stack Position
}

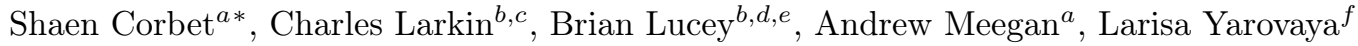 \\ ${ }^{a}$ DCU Business School, Dublin City University, Dublin 9 \\ ${ }^{b}$ Trinity Business School, Trinity College Dublin, Dublin 2 \\ ${ }^{c}$ Institute for Policy Research, University of Bath, UK \\ ${ }^{d}$ University of Sydney Business School, University of Sydney, Sydney, New South Wales, Australia \\ ${ }^{e}$ Distinguished Research Fellow, Institute of Business Research, University of Economics Ho Chi Minh City, 59C \\ Nguyen Dinh Chieu, Ward 6, District 3, Ho Chi Minh City, Vietnam \\ ${ }^{f}$ Southampton Business School, University of Southampton, Southampton, SO17 1BJ, UK \\ *Corresponding Author: shaen.corbet@dcu.ie
}

\begin{abstract}
We examine the response of a broad set of digital assets to US Federal Fund interest rate and quantitative easing announcements, specifically examining associated volatility spillover and feedback effects. We classify each digital asset into one of three categories: Currencies; Protocols; and Decentralised Applications (dApps). Currency-based digital assets experience idiosyncratic spillovers in the period immediately after US monetary policy announcements, while application or protocolbased digital assets are largely immune to policy volatility spillover and feedback. Mineable digital assets are found to be more susceptible to monetary policy volatility spillovers and feedback than non-mineable. Responses indicate a diverse market within which, not all assets are comparable to Bitcoin.
\end{abstract}

Keywords: Cryptocurrencies; Digital Assets; GARCH; Volatility Spillovers; Monetary Policy.

\section{Introduction}

Bitcoin is proposed as a decentralised currency requiring no third-party involvement, and independent of central banks and governments. Despite the current position of Bitcoin as the cryptocurrency market leader, it will experience increased competition in the near future, as the blockchain space matures. A number of studies suggest that legitimate challengers to Bitcoin will emerge (Fry and Cheah [2016]). The majority of research has focused solely on Bitcoin, with various other alternative-coins being relatively ignored, however, there has been a recent emergence of significant competitors to Bitcoin (for example, Ethereum and Litecoin) and this will only continue to increase as cryptocurrencies with improved technology and increased efficiency (such as EOS, Tezos and Factom) continue to emerge. The creation of new blockchain protocols looks set to surpass Bitcoin 
as the sole major cryptocurrency, and it has already been noted that the emergence of such coins has a negative impact of the growth of Bitcoin.

The current internet stack is comprised of a number of shared protocols (such as HTTP, TCP and SMTP) on top of which an application layer is built. The application layer is comprised of the centralised web applications that make up the Internet as we know it (utilised by online services such as Gmail, Facebook and Airbnb). Although consumers and end-users are typically only familiar with the end applications, such applications and indeed the whole internet, would not be possible without these underlying protocols. Lower-level protocols will be necessary to provide infrastructure that will allow user-friendly decentralised applications to be built. Financial cryptocurrencies that are intended solely for the transfer of wealth and to be used as payments systems, are just one of the possible applications on the top layer of the blockchain stack. While previous studies have primarily focused on Bitcoin itself, the emergence of blockchain-based tokens necessitates further study to determine the key differentials between these different forms of tokens, and to assess whether they react differently to the market. We observe a distinct difference between the two layers described as protocols and applications. Our research aims to quantify this theoretical difference by measuring their respective reactions to fiat money monetary policy (i.e. monetary policy related to financial instruments related to a denominated legal tender as defined in law by national and supranational governments).

We note key differences among application layer tokens themselves, and the large number of varying forms that currently exist. As currency based tokens currently dominate and account for most of the current market, in terms of market cap and public awareness, it is appropriate to denote these tokens as a separate asset class. Having classified currency based digital assets as their own category, we view all other applications in the top layer as one category. As the digital assets market is currently at a very early stage of development, it is likely that these categories and classifications will change as new use cases are developed. We aim to differentiate between assets used at the bottom layer of infrastructure, that will primarily be of use to developers over the coming years, and top layer applications that will be intended for use by the wider public. Again, with regard to these applications, we differentiate between those that are intended for use primarily as currency and financial transfer and those that have any other use case. We classify each digital asset in one of three categories:

1. Currencies: Digital assets whose primary (and in most cases, only) use is that of financial payment or monetary transfer.

2. Blockchains/Protocols: Digital assets whose primary usage is that of a blockchain platform, or protocol, on which other applications can be built.

3. Decentralised Applications (dApps): Applications combining a user interface, and a decentralised back-end, built upon an already existing blockchain.

Using a range of GARCH-family methodologies, we examine the interlinkages of individual digital assets to US monetary policy changes, in the form of interest rate and quantitative easing (QE) announcements. We identify volatility spillover transfers between these policy changes and the digital assets market as a whole, before dividing our sample into three, separated by type of cryptocurrency (Currency, Protocol and dApp) and re-examining volatility spillover transfer to each of the three categories. We examine the differing reaction between mineable and non-mineable assets, before using a second-stage GARCH model to identify any changes in volatility spillover characteristics as the digital assets market matures. It is important to determine whether there exists traditional volatility spillover channels between broad financial markets and this new asset 
class. We observe a statistically significant differentiation between applications, and the protocols upon which they are built. We find evidence of volatility spillover transfer from US monetary policy announcements to Currency-based digital assets. In contrast, Application or Protocol-based digital assets remained largely immune to policy spillover transfer. Additionally, we find evidence to suggest that mineable digital assets are more susceptible to monetary policy volatility spillovers transfer than non-mineable. We also find evidence to suggest that Currencies experience a positive increase in global systematic volatility spillovers (following a monetary policy announcement) while Protocols experience a decrease. dApps remain immune to any global systematic volatility spillover transfer, throughout our sample.

\section{Previous Literature}

Cryptocurrency research has developed across a broad number of areas, with specific emphasis on pricing inefficiencies (Sensoy [2019], Corbet et al. [2019]), to be in isolation from other traded assets (Corbet et al. [2018]), to present evidence of price clustering (Urquhart [2017]), pricing bubbles (Corbet et al. [2018]), regulatory ambiguity (Fry [2018]), and exceptional levels of both complex and uncomplex fraud (Gandal et al. [2018]). Although development and innovation within financial markets is of course associated with some risk, due to the exceptional number of relatively illicit activity within the product's pricing, trading techniques and indeed the exchanges on which it trades, the product's development has continued at pace, despite the exceptional pricing volatility that has taken place (Katsiampa et al. [2019a]; Katsiampa et al. [2019b]; Celeste et al. [2019]). Due to the relative youth of cryptocurrency markets, we must draw on a number of sources of prior research that focus on broad symptoms of market stress. Berger and Bouwman [2017] examined the interplay among bank liquidity creation, monetary policy, and financial crises, where results suggest that authorities may wish to monitor bank liquidity creation closely in order to predict and perhaps lessen the likelihood of financial crises. Christopoulos and Jarrow [2018] investigated CMBS market efficiency during and after the credit crisis with a comprehensive loan, bond and deal level data set, while Yoldas and Senyuz [2018] constructed models that incorporate the long-run equilibrium relationship between term Libor and OIS rates and their regime-dependent dynamics to find strong evidence for three regimes in the inter-bank funding market that resemble different pricing of risk and equilibrium outcomes. Development of policy and regulatory solutions to issues relating to volatility and interactions in such maturing markets are central to the development of policy (such as that of Mester [2017]; Krainer [2017]; and Gertler and Horvath [2018]) from which cryptocurrency markets can further progress. Evidence of such market maturity has been identified in works such as that of Akyildirim et al. [2019], who found that there have been broad, significant pricing effects sourced from both fraudulent and regulatory unease within the cryptocurrency industry. A concise overview of associated cryptocurrency literature was developed by Corbet et al. [2019]. Our work delves deeper into the specific questions surrounding the differences in behaviour with regards to sub-categories of cryptocurrencies. Eken and Baloglu [2017] compared the development of cryptocurrency markets with other currency types that were in use in different periods of history. Dwyer [2015] provides an explanation of the way in which digital currencies, such as Bitcoin, use peer-to-peer networks to overcome the double-spending problem, referring to an error in a digital cash scheme in which the same single digital token is spent more than once ${ }^{1}$. This is possible because

\footnotetext{
${ }^{1}$ In decentralised systems, by 2007, a number of distributed systems for double-spending prevention had been proposed. Bitcoin implemented a solution in early 2009, using a scheme called 'proof-of-work', to avoid the need
} 
a digital token consists of a digital file that can be duplicated or falsified. As with counterfeit money, such double-spending leads to inflation by creating a new amount of fraudulent currency that did not previously exist. This devalues the currency relative to other monetary units, and diminishes user trust as well as the circulation and retention of the currency ${ }^{2}$.

Böhme et al. [2015] provide a detailed description of the technology behind Bitcoin, including the blockchain, mining, mining pools, transaction fees and wallets. The authors also detail the early use cases of Bitcoin, areas of risk involved, and examine the potential for future regulation. Kroll et al. [2013] examine the economics of Bitcoin mining and mining strategy, in particular, its resilience to potential attacks. The authors observe the presence of a Nash equilibrium which requires players to reach a consensus on three key factors: 1)The rules: how to determine a valid transaction; 2) The state: an agreement on the history of the blockchain; and 3) The value of Bitcoin (participants must be in agreement that Bitcoin is inherently valuable). The authors highlight Bitcoin's vulnerability to various potential attacks, including a ' $51 \%$ ' attack (an attack by a participant who controls more than $51 \%$ of the network power) and a Goldfinger attack (an attack motivated by a desire to disrupt the system, due to an outside incentive. Potential attackers include: law enforcement, a holder of a short position and protesters).

There have been multiple pieces of research that utilise GARCH-family methodologies to specifically investigate cryptocurrency pricing behaviour. Gronwald [2014] analyse Bitcoin pricing behaviour, identifying that Jump (ARJI) GARCH models are found to outperform GARCH(1,1) models, finding evidence that cryptocurrency markets are behaving in a very immature manner when compared to traditional markets. Risteski and Davcev [2014] extended the conventional EGARCH model with modification through the addition of Search Volume Index (EGARCH-SVI) as measured by Google Trends data. Brandvold et al. [2015] examine the varying price of Bitcoin among different exchanges and the contribution of these exchanges to price discovery. Mt Gox and Btc-e are found to be the price leaders. Smaller exchanges are found to provide the market with less information, and usually follow the market with a lag. Akyildirim et al. [2019] identified the existence of time-varying positive interrelationships between the conditional correlations of cryptocurrencies and financial market stress when analysing the relationship between the price volatility of a broad range of cryptocurrencies and the VIX and VSTOXX respectively. Bouoiyour and Selmi [2016] examined the 2016 decline in the volatility of Bitcoin and ask whether this is an indication that the Bitcoin market is maturing. Using multiple-threshold and asymmetric-power GARCH models, the authors compare returns over two periods (2010-2014 and 2015-2016). The authors determine 2010-2014 to have been an 'explosive process' and observe Bitcoin volatility persistence to have fallen in 2015. In addition, the authors find Bitcoin prices to be asymmetrically driven. Bariviera et al. [2017] compare dynamics of Bitcoin and traditional currency returns. The authors focus on the long-range memory of returns and find Bitcoin volatility to be reducing over time.

for a trusted third party to time-stamp transactions. These time-stamps are recorded in its public ledger called the blockchain, therefore it avoids double-spending of the currency. The use of this method, combined with a limitation of supply, leads to an equilibrium. Additionally, the author notes the rise of $24 / 7$ online trading markets without the need for any brokers or agents and determines Bitcoin to have a comparative advantage over traditional fiat currencies, due to the low associated transactions costs and the potential for avoidance of government currency controls

${ }^{2}$ Fundamental cryptographic techniques to prevent double-spending while preserving anonymity in a transaction are blind signatures and particularly in off-line systems. In centralised systems, this is usually implemented using an on-line central trusted third party that can verify whether a token has been spent. This normally represents a single point of failure from both availability and trust viewpoints. 
Additionally, Bitcoin dynamics are found to have changed over time and, using the Hurst exponent, Bitcoin is found to have stabilised in recent years.

Analysis of the volatility behaviour of these products is vital when considering the broad range of pricing anomalies and bubble-like pricing behaviour that has been supported with evidence. Cheah and Fry [2015] test for evidence of speculative bubbles in Bitcoin returns, using two assumptions for intrinsic rate of return and intrinsic level of risk. The authors find evidence to suggest that Bitcoin prices are prone to substantial bubbles. In addition, they empirically estimate the value of Bitcoin to be zero. Urquhart [2017] measures the efficiency of Bitcoin returns over a six-year period (between August 2010 and July 2016) using a number of tests for randomness (Ljung-Box, Runs, Bartels, Automatic variance test, BDS, R/S Hurst tests), finding that returns are significantly inefficient over the entire sample. When divided into two equal sub-samples, two tests indicate efficiency of returns in the latter sample, suggesting that Bitcoin may be moving towards becoming more efficient. Nadarajah and Chu [2017] elaborate on this study by using a power transformation of the same returns used by Urquhart [2017], without any loss of information. Having performed this transformation, returns displayed evidence of weak efficiency over both the entire sample, and both sub-samples. Cheung et al. [2015] perform an econometric investigation of bubbles in the Bitcoin market, using the Phillips et al. [2015] methodology (a technique which has proven to be robust in detecting bubbles). Using this method, the authors detect a number of short-lived bubbles, and three large bubbles lasting from 66 to 106 days. The bursting of these bubbles is found to coincide with a number of major events that occurred in the Bitcoin market, the most significant of these leading to the demise of the Mt. Gox exchange (as a result of the Chinese ban and the theft of a large amount of Bitcoin). Further evidence has identified the presence of substantial bubbles in a range of cryptocurrency markets (Corbet et al. [2018]; Fry [2018]).

\section{Blockchain Positioning}

Blockchain based cryptocurrencies are protected by Merkel Trees, a cryptographic method that prevents the blockchain from being altered, once a block has been confirmed (the more subsequent blocks that are added, the harder it becomes to alter previous blocks). A blockchain consists of a series of data packets, intrinsically linked to one another, known as blocks. Each block is added to the chain by a series of computations completed by a network of users. Each block is added to the existing chain, in such a way that it becomes near impossible to alter the data that has been entered in previous blocks. This enables transactions, and other data, to be stored online, and enables users to have full confidence that the data cannot be tampered with, or changed, at a later date. Such a system allows peer-to-peer monetary transactions to become possible online, by overcoming the previously insurmountable double-spending problem.

Traditional fiat currency, gold, and other stores of value derive value due to their scarcity. For digital currencies to possess value, they must be scare, a shortcoming of all previous attempts at creating digital currencies. The mining process creates, and ensures scarcity. Thousands of copies of the blockchain are stored and run on computers around the world, known as nodes. All transactions that occur within the network are recorded, and kept track of, in a ledger (the blockchain). Previously, it would fall upon central banks to maintain the ledger, and ensure that no egregious changes, or forgeries. are made. As opposed to centralised banks maintaining control of the ledger, as is the case with traditional fiat currencies, the ledger is maintained by each of the nodes. Nodes communicate with each other to determine what the correct form of the ledger is. Consensus is arrived at, between these nodes, as to which copy of the ledger is the correct 
one and, as a result, the need for a centralised third-party validator is removed. Full nodes verify and maintain the ledger, in exchange for economic incentives: miner rewards and transaction fees. Nodes compete with each other, in an attempt to solve a complex mathematical problem, which allows for the confirmation of the next block onto the chain. Miners allocate resources, in the form of computing power, to this pursuit, in the hope of receiving a block reward (a payment of Bitcoin to the first node to arrive at the correct solution to the mathematical problem). Digital assets derive their value, and the scarcity necessary for value, from this mining process. Digital assets can be stored using a variety of methods: online wallets, online exchanges, hardware wallets and paper wallets (cold storage) being just a selection of the possible storage methods.

Smart contracts enable the transfer of traditional real-world agreements, onto the blockchain, so that they can be executed online - without the need for a third-party enforcement agent. Smart contracts are computer protocols, intended to facilitate, verify or enforce the negotiation or performance of a contract. Smart contracts define the rules and requirements around a proposed agreement, in the same way that a traditional contract does, however, the smart contract automatically enforces the obligations, removing the need for a trusted third-party.

\section{Insert Table 1 about here}

The protocol layers that enable, and define, internet communication are collectively referred to as the internet protocol stack. Figure 1 presents an illustration of the TCP/IP stack. The lowest, link layer, allows for the transfer of data packets between different hosts on a local network. Hardware protocols such as Ethernet allow for the transfer of data over LANs (Local Access Networks), although the TCP/IP protocol can be implemented on top of almost any hardware. The internet layer allows these data packets to be sent across multiple networks through a routing process. Internet layer protocols such as IP (Internet Protocol) carry data for a number of upper-layer protocols. The transport layer allows for end-to-end communication across a network between application processes running on different networks. Such communication is facilitated through a connection-orientated protocol such as TCP (Transmission Control Protocol). The application layer consists of the necessary protocols - such as HTTP (Hypertext Transfer Protocol) and SMTP (Simple Mail Transfer Protocol) - required for common applications to send and receive application data across the lower level protocols, enabling it to provide a service for the user. The combination of these layers is referred to as the protocol layer, and is the necessary foundation that common web applications such as Gmail and Google are built upon. The blockchain market and ecosystem should be viewed in the same manner, not as a series of individual currencies, but as a blockchain stack. Cryptocurrencies are frequently viewed by investors as one and the same, but there are large differences between the various categories that these cryptocurrencies fall under. In the same way that Gmail is a single application of the entire Internet stack, and the protocols that underlie it, Bitcoin is a single use of its underling protocol, the blockchain. Blockchains enable the transfer and sharing of data to take place in a decentralised, and transparent, manner. These blockchains should be viewed similarly to HTTP and TCP - as the bottom layer of the blockchain stack. Only once these protocol layers have been sufficiently developed, can user-friendly applications be built upon them. Contrary to public opinion, the blockchain's use is not limited to purely facilitating financial transfers and transactions. Blockchains enable the transfer of data, and so, allow a number of possible use cases to be built upon them. Such uses include, file and data sharing (IPFS, Filecoin), decentralised super-computers (Golem), and the execution of smart contracts, first demonstrated by 
Ethereum. As such, it would be inappropriate, and incorrect, to compare and contrast all tradeable tokens in the digital asset market, with Bitcoin. Bitcoin is merely a single use case of a much wider market. A visual representation of a potential blockchain stack is also provided in Figure 1.

The current digital assets market currently consists of a number of tokens from both the protocol and application layers of the blockchain stack - a dynamic not previously observed in the early days of the internet. Such a dynamic is created due to the newfound ability of blockchain protocols to create wealth. Shared internet protocols such as TCP/HTTP are essential to the modern internet, however they generate no value for users and it falls upon the top-end applications to do so. In the case of the blockchain stack, low-level protocols such as Ethereum, can be monetised - through the creation of a token - and so, are capable of generating wealth. As illustrated by Figure 1, these protocols will be capable of generating large amounts of wealth, as they are an essential component of every top-level application built upon them. As such, we consider it incorrect to view the digital assets market as one entity - similar and comparable to Bitcoin - and instead, suggest that assets should be evaluated based on their position within the blockchain stack.

1. Currencies: The most notable, and widely used, digital asset is Bitcoin. Bitcoin is a peer-topeer digital asset, which claims to be decentralised and independent of monetary authority influence, created by Satoshi Nakamoto (Nakamoto [2008]). Transactions take place directly between users, and are verified by network nodes. To overcome the traditional 'doublespending' problem, each transaction must be cryptographically verified by a network node, or 'miner'. Kroll et al. [2013], provides a detailed description of the mining process). Miners add verified transactions to a publicly distributed ledger, or blockchain, and are incentivised to do so by the reward of transaction fees and new bitcoins. New bitcoins are created every 10 minutes, via this mining process, and will continue to do so until the full supply, of 21 million bitcoins, has been issued. In this way, unlike traditional FIAT currencies, Bitcoin is a deflationary asset. As the digital assets market has expanded, a number of other peer-to-peer currencies have emerged. Some have made minor adjustments and changes to the original Bitcoin source code, while others have created an entirely new asset. Alternative coins, or altcoins, typically aim to improve upon Bitcoin in areas such as privacy, transaction speed and cryptographic proof method. We define any digital asset who's primary function is that of a medium of exchange or transfer of value, as a Currency.

2. Protocols: We define a protocol as a set of rules governing the transfer of data across networks. Figure 1 contains a number of examples of internet protocols including: Hypertext Transfer Protocol (HTTP), the underlying protocol of the World Wide Web defining how messages are formatted and transmitted; HTTP Secure (HTTPS), a communications protocol used for secure communication over networks; and Secure Sockets Layer (SSL) a communications security protocol commonly used in email. Unlike Currencies, we define blockchain Protocols as digital assets whose primary function will be that of data transfer, and provide a bottom layer on which to build decentralised applications. When categorising the digital assets in our sample, we include any platform, blockchain or method for data transfer, upon which other applications could feasibly be built, in our definition of the term 'protocol'.

3. Decentralised Applications (dApps): The term decentralised application (dApp) refers to any application whose back-end code runs on a decentralised peer-to-peer network. This contrasts with traditional applications, whose back-end code is usually executed on a centralised server. dApps typically consist of front-end code and user interfaces written in a traditional programming language, and back-end code executed on a blockchain - typically in the form of 
smart contracts. For the purposes of this study, we define dApps as any decentralised application built upon a blockchain or platform, whose primary purpose is not that of currency or monetary transfer. Examples of blockchain dApps include decentralised storage applications, insurance applications, and decentralised autonomous organisations (DAOs).

We define a digital asset as any asset in binary format owned by an individual. For the purpose of this study, we collectively refer to the combination of cryptocurrencies, blockchain platforms, protocols and decentralised applications contained in our sample, as digital assets. A coin such as Bitcoin, is a single application built upon its underlying blockchain. A protocol such as Ethereum allows smart contracts to be executed upon its blockchain, and in doing so, allows decentralised applications to be built upon it. Any application built upon a blockchain, is intrinsically linked with that blockchain. A token issued upon the Ethereum blockchain (referred to as an ERC-20 token) requires Ether to execute the smart contracts that allow this token/application to function. In this way, as the token grows, so does the underlying protocol, and any wealth generated at the application layer, is distributed not only among its own token holders, but back to the underlying protocol itself. As such, Ethereum token holders benefit not only from the growth of Ethereum, but from the growth of any application built upon the platform - as a growth in the token, inherently causes the platform itself to grow. As such, when attempting to price or economically analyse such assets, it would be inappropriate to compare a token with the blockchain that token itself is built upon, the two should be viewed as entirely different classes of asset.

\section{Data and Methodology}

\subsection{Data}

Unlike traditional equities and commodities, digital assets are traded on a number of exchanges; as such, closing prices can vary depending on the exchange used to source the data. Data is obtained from www.coinmarketcap.com, where closing prices are constructed using a weighted combination of closing prices from all exchanges on which the asset is traded.

\section{Insert Table 1 about here}

We source daily closing prices for all assets, and exclude any assets that provide less than 90 days of observations, leaving us with a remaining 58 digital assets in our sample displayed in Table 1. We use the US nominal broad dollar index, a trade-weighted index used to measure the value of the dollar, relative to other world currencies to represent a global currency factor. We identify Bitcoin as the market leader among digital assets. Due to its large market capitalisation, relatively stable price, and high level of trust and reputation among users as the market leader, we use Bitcoin returns as a proxy for a domestic (US) currency factor. We refer to each variable in our sample (digital assets and currency pairs) as an asset.

\section{Insert Table 2 about here}

We aim to examine the reaction of each asset in our sample to US policy announcements. To do this, we construct a database of US policy changes between the 26th April 2013 and the 30th June 
2017. Our announcement database consists of eight interest rate changes and quantitative easing (QE) announcements made by the US Federal Market Open Committee, between May 2013 and June 2017, and is presented in Table 2. We denote the 50 days following the announcement of an interest rate or QE change, as the announcement period.

\section{Insert Table 3 about here}

We sort our sample by type of cryptocurrency, denoted as either a Currency, Protocol or Decentralised Application (dApp), based on the definitions provided in previous sections of this paper. To summarise: we denote a Currency as any digital asset whose primary use is monetary payments, currency related, or a store of financial value. We denote a Protocol as any digital asset whose primary function is that of a blockchain protocol or platform. We denote a dApp, as any third-party application, with a primary function other than currency, built on an existing blockchain.

\subsection{Methodology}

We note the presence of heteroscedasticity and volatility persistence (Andersen and Bollerslev [1997]) in our returns data. When testing for contagion and volatility spillovers, methods which do not correct for heteroscedasticity are found to be biased (Forbes and Rigobon [2002]) - such tests will overstate any increases in market volatility, and the magnitude of cross-market relationships. As such, non-heteroskedastic adjusting tests may incorrectly suggest that volatility spillovers have occurred. To account for this, we implement a variation of the generalised autoregressive conditional heteroscedasticity (GARCH) based approach (Edwards [1998] and Engle et al. [1990]). Specifically, we use the exponential generalised autoregressive conditional heteroscedasticity (EGARCH) model, as it allows for asymmetric effects between positive and negative returns. After completing the standard robustness tests the $\operatorname{EGARCH}(1,1)$ methodology, for the most part, was selected as the appropriate model to test for changes in volatility ${ }^{3}$. Where necessary, we display the results of the best-fitting methodology as determined by the selection criteria ${ }^{4}$ displayed in Table 3 . Models that incorporate volatility asymmetries, or negative correlations between returns and volatility innovations, generally outperform models that do not. Further, the EGARCH methodology exploits information contained in realised measures of volatility while providing a flexible leverage function that accounts for return-volatility dependence while remaining in a GARCH-like modelling framework and estimation convenience, the model allows independent return and volatility shock and this dual shock nature leaves a room for the establishment of a variance risk premium. We estimate the standard market model as follows:

$$
r_{i, t}=a_{0, i}+a_{1, i} f_{t}^{\text {global }}+\epsilon_{i, t}
$$

\footnotetext{
${ }^{3}$ We considered the use of GARCH, Threshold GARCH (TGARCH) and GJR-GARCH, but EGARCH was found to outperform each methodology. Results are available from the authors on request. An intercept and a deterministic trend were included in the Augmented Dickey Fuller (ADF) and Phillips Perron (PP) models. The trend was included to capture the reduction in average volatility that took place during the period analysed. The ADF model tests whether the equity series contain a unit root in order to correct for serial correlation. PP tests employ a nonparametric estimator of the variance-covariance matrix with $d$ truncation lags. The models test down by sequentially removing the last lag until a significant lag is reached giving the order of augmentation for the ADF test that minimised the Akaike information criterion. The results indicated rejected of the null-unit root hypotheses at a minimum of the 5 per cent level

${ }^{4}$ Results for all methodological calculations are available from the authors on request.
} 
where $f^{\text {global }}$ represents the global currency factor in the form of the US broad dollar index, representing the common shock to all currencies. $a_{(1, i)}$ is a measure of global systematic risk exposure for asset i. $r_{(i, t)}$ represents the return of asset i at time t. To identify systematic volatility spillovers, we extend specification (1) as follows:

$$
r_{i, t}=a_{0, i}+a_{1, i} f_{t}^{\text {global }}+a_{2, i} f_{t}^{\text {global }} I_{t}+\epsilon_{i, t}
$$

$I_{t}$ takes the value 1 during an announcement period, and 0 during all other periods. $a_{2, j}$ therefore, is a measure of the change in systematic risk exposure following the interest rate announcement, and can be used to represent systematic volatility spillovers. To capture both idiosyncratic and shift volatility spillovers, we extend the specification as follows:

$$
r_{j, t}=a_{j, 0}+b_{1, j} f_{t}^{\text {global }}+b_{2, j} f_{t}^{\text {global }} I_{t}+b_{3, j} f_{t}^{B T C}+b_{4, j} f_{t}^{B T C} I_{t}+\xi_{i, t} ;
$$

where, $j=1, \ldots \ldots, n-1 \neq U S . \quad f_{t}^{B T C}$ represents the US domestic currency factor, proxied by Bitcoin returns. $b_{1, j}$ represents a standard CAPM beta coefficient against global markets. $b_{2, j}$ represents systemic volatility spillovers (as previously discussed). $b_{3, j}$ measures any spillover effects that occur as a result of shocks emanating from the interest rate announcement. $b_{4, j}$ is a measure of the change in spillover effects that occurred solely during an announcement period. These additional spillover effects then, can be used to denote idiosyncratic volatility effects. $b_{5, j}$ captures any intercept shift in the model that occurred during the announcement period, and is used to represent shift volatility spillovers. We express the variance equation of our EGARCH model as follows:

$$
\begin{aligned}
\ln \left(\alpha_{j, t}^{2}\right)= & c_{0, i}+c_{1, i}\left(\left|Z_{i, t-1}\right|-E \mid Z_{i, t-1}\right)+c_{2, i} z_{i, t-1}+c_{3, i} \ln \left(\alpha_{i, t-1}^{2}\right) \\
& z_{i, t-1}=\frac{\eta_{i, t-1}}{\alpha_{i, t-1}} ; \eta_{i, t}=\left\{e_{i, t-1}, \eta_{i, t-1}, \xi_{i, t-1}\right\} \\
& \eta_{i, t} \sim \text { student }_{t}\left(0, \sigma_{i, t}^{2}\right)
\end{aligned}
$$

We use the following variance equation to capture the volatility spillover effects emanating from the US, which we use to identify volatility volatility spillover:

$$
\begin{aligned}
\ln \left(\sigma_{j, t}^{2}\right)= & c_{0, j}+c_{1, j}\left(\left|Z_{i, t-1}\right|-E \mid Z_{i, t-1}\right)+c_{2, j} z_{j, t-1}+c_{3, j} \ln \left(\sigma_{j, t-1}^{2}\right) \\
& +\pi_{1, j} \ln \left(\sigma_{B T C, t}^{2}\right)+\pi_{2, j} \ln \left(\sigma_{B T C, t}^{2}\right) I_{1, t}+\pi_{3, j} \ln \left(\sigma_{B T C, t}^{2}\right) I_{2, t} \\
& \text { where } ; j=1, \ldots \ldots, n-1 \neq B T C
\end{aligned}
$$

$\pi_{1, j}$ represents general US volatility spillover, over the entire sample period. $\pi_{2, j}$ measures the US volatility spillover that occurred during the announcement period itself. This measure of additional volatility spillover is used to represent volatility driven spillover.

\section{Results}

\subsection{Volatility spillover and feedback effects}

Using our previously outlined methodology, we find significant evidence of volatility spillover transfers from US monetary policy announcements, to the digital asset market. With the exception 
of four, relatively small-cap currencies, all other digital assets in our sample exhibit statistically significant evidence of exposure to global systematic risk during the sample period. The four exceptions (Gulden, ReddCoin, Xaurum and CloakCoin) are all relatively small-cap assets, and are all classified as Currencies (Gulden, ReddCoin and CloakCoin are all used for payments, while Xaurum represents a store, or unit, of value).

A digital asset's change in exposure to global systematic risk, as a result of the monetary policy announcement, is represented by the coefficient $b_{2}$. An increase in exposure to global systematic risk, in the period following the policy announcements, indicates systematic volatility spillover generated solely by the monetary policy announcement. All digital assets which experienced exposure to systematic volatility spillover were either Currencies or Protocols. We observe no evidence of systematic volatility spillover in any of the dApps in our sample. A total of twelve digital assets experienced systematic volatility spillover exposure. Of this total, seven experienced a significant increase in systematic volatility spillover, while five experienced a significant decrease. The seven digital assets which experienced an increase (Bytecoin, BitcoinDark, Burst, Leocoin, Monacoin, ReddCoin and Stellar Lumens) are all classified as Currencies. Four of the five assets which experienced a decrease (AntShares, Ethereum, NXT and Stratis) were classified as Protocols. Peercoin is the only currency in our sample to have experienced a significant increase in global systematic volatility spillovers. Of the five assets which displayed evidence of negative exposure to global systematic volatility spillovers, all five had previously displayed positive exposure with the global market, prior to the monetary policy announcements.

The unreported coefficient, $b_{3}$, represents the general spillover effects Masson [1999] that emanate from the domestic cryptocurrency market, represented by Bitcoin (BTC). The coefficient $b_{4}$ then, represents the additional BTC spillovers that occurred, following each monetary policy announcement, and we denote this effect as idiosyncratic volatility spillover. Almost all of the assets in our sample displayed evidence of exposure to idiosyncratic volatility spillover ( $82 \%)$. The ten assets which display no evidence of such spillovers (AntShares, Mona, Synero, PivX, Status, Monero, Verge, ReddCoin, Cloak and NEM) are a combination of all three classifications of asset. Only Ethereum and Bytecoin display a negative, statistically significant, idiosyncratic spillover coefficient. The presence of shift volatility spillover is represented by the coefficient $b_{5}$. Almost half (47\%) of our sample experienced shift volatility spillover. Of the assets to have experienced shift volatility spillover, fifteen were Currencies, eight Protocols and five dApps.

\section{Insert Table 4 about here}

As illustrated in Table 4, exactly half of the digital assets in our sample (29) exhibited some form of US monetary policy generated volatility spillovers. A small group of four digital assets (CloakCoin, EarthCoin, Syscoin and NEM) exhibit volatility spillover effects that were driven by volatility spillovers. Three of these assets are relatively small assets, with the exception of NEM, a large-cap protocol (market capitalisation of $\$ 1.7$ billion, the sixth largest in our sample). A small group of five assets (Agoras Tokens, Gulden, ReddCoin, Xaurum and Stellar Lumens) display evidence of effects that were primarily driven by systematic volatility spillovers. With the exception of Agoras, the remainder are all classified as a Currency. Again, the majority of the assets are relatively small-cap, excluding Stellar Lumens (\$286 million market capitalisation, the 26th largest). Ten assets display evidence of spillover effects that were driven by idiosyncratic shocks. This group is primarily comprised of Currencies, indicating that it was this category which 
was most likely to experience the effects of monetary policy generated shocks. Additionally, this group features a number of the largest assets in our sample (Bitcoin, Litecoin and Dash) suggesting that, as a digital asset which is primarily used for financial payments and monetary transfers becomes larger, it becomes more exposed to traditional currency monetary policy announcements. A further ten assets experienced multiple drivers of volatility spillovers. Again, the list is primarily comprised of Currencies and Protocols. Notably, Ethereum (a Protocol, and the second largest market-cap in our sample) displays evidence of such exposure to multiple forms of spillovers. The lack of any dApps on this list is notable, and the fact that only two in total experience volatility spillover driven effects of any kind, suggests that this category remains predominantly immune to US monetary policy effects.

A total of $76 \%$ of Currencies in our sample experienced some form of volatility spillover. When compared with the presence of Protocols which experienced a form of spillover transfer (40\%), it is clear that digital assets which are primarily used for monetary transfer and financial services, are more susceptible to US monetary policy volatility spillovers transfer. The fact that only $20 \%$ of the dApps in our sample experience any form of volatility transfer, suggests that this category of assets remain immune to traditional currency monetary policy effects. Having examined the volatility spillover responses of our entire sample to policy decisions, we now segregate our sample based on 1) category and 2) whether the digital asset is mineable or not. After separating by type of cryptocurrency, we re-estimate our results using the same methodology contained in the previous section.

\subsection{Behavioural differences between currencies, protocols and dApps}

The three groups of cryptocurrencies contain 28, 20 and 10 digital assets, respectively. All three categories display evidence of positive exposure to global systematic risk, confirming the previous sections result, and indicating that digital assets, as a whole, are subject to global market risk exposure. While a number of individual assets experienced exposure to systematic volatility spillovers, once separated into groups, none of the three categories display significant evidence of such exposure. The fact that affected individual assets are all predominately large-cap suggests that, as an asset's market capitalisation increases, it becomes significantly exposed to global systematic spillovers, while the digital asset market as a whole remains mostly immune. Whether or not this increased exposure is positive or negative depends on the category of asset. The previously identified split between positively and negatively exposed assets, dependent on their being Currencies or Protocols, suggests that, as a Currency increases in market cap, it become more exposed to systematic volatility spillovers, while Protocol layer assets will experience reduced exposure.

Idiosyncratic volatility spillovers are found to be positive, and statistically significant, in both the Currency and dApps groupings. The Protocol cohort displays no significant evidence of exposure to this form of spillover. Having previously presented evidence of idiosyncratic spillover in $82 \%$ of the total sample, this becomes an important result. Protocol level digital assets clearly display a reduced propensity for idiosyncratic volatility spillover exposure, when compared with other assets in the market. This finding is a notable contribution to the quickly expanding area of digital assets and cryptocurrencies and confirms, for the first time, that all digital assets should not be viewed as having similar market characteristics. Digital assets, in fact, react differently to traditional markets and policy announcements, based on the specific use of the asset. Digital assets have largely been compared to, and associated with Bitcoin, due to their relative complexity and the lack of research on the area. The fact that Protocol layer digital assets remain immune to US monetary policy spillovers confirms that each asset should be evaluated and valued based on its own individual use, 
rather than be viewed as one entire 'Bitcoin-like' market. Having previously identified exposure to shift spillovers in half the total sample, the presence of positive and significant exposure to shift spillovers, in each of the three groups, confirms that this form of volatility spillover is evenly distributed amongst all forms of asset.

\section{Insert Table 5 about here}

As further illustrated by the parameter results in table 5, just one of the three cohorts displays evidence of volatility spillover effects that were driven by US monetary policy announcements. Currency volatility spillover effects are shown to be driven by idiosyncratic shocks, a result which is consistent with the findings of Section 4, in which the group of individual assets to be idiosyncratically driven, consists predominantly of larger-cap Currencies. Neither Protocols nor dApps displayed evidence of having any volatility driven effects, a result which is also consistent with earlier presented findings. Just $40 \%$ of individual Protocols, and an even smaller $20 \%$ of dApps, displayed evidence of having some form of US policy driven spillover effects. Neither of these groups displays significant evidence of such spillover driven effects, indicates that they remain largely immune, and considerably less effected, when compared to Currencies. This result is both significant, and intuitive, suggesting that digital assets whose primary purpose is that of fiscal use and financial transfer, are susceptible to the same US monetary policy generated market volatility spillovers as traditional currencies, while digital assets created for other purposes, remain immune to such policy measures.

\subsection{Behavioural differences between mineable and non-mineable assets}

We further divide our entire sample into two additional cohorts: assets which are mineable, and those which are not. The two groups contain 30 and 28 digital assets, respectively. We note significant differences between the two groups with regard to the $b_{1}, b_{2}$ and $b_{4}$ coefficients. In Table 6, we observe that mineable assets display evidence of significant positive exposure to global systematic risk, consistent with our results from both previous models. However, non-mineable assets are shown to be an exception to this rule, and display no significant evidence of exposure to such risk.

\section{Insert Table 6 about here}

In the case of exposure to systematic volatility spillovers, mineable assets display a negatively significant change in systematic risk exposure following policy announcements, indicating exposure to systematic spillovers. Again, in contrast, non-mineable assets show no such evidence of exposure. We previously noted a lack of significant systematic volatility spillovers in any of the three categories, however, when viewed individually we noted that a number of currencies and protocols experienced a significant increase or decrease in this form of spillover exposure. This observation is explained by the increased likelihood of a Currency or dApp being mineable, when compared to a Protocol or blockchain itself. In the case of the original twelve assets that experienced systematic exposure - seven were mineable assets. Such results clearly highlight and confirm an increased propensity for systematic volatility spillover exposure in mineable assets. The $b_{4}$ coefficient, representing additional BTC spillovers that occurred following a policy announcement, is found to be 
significant, and negative, in the case of the group of mineable assets. Once again, we observe a non-significant coefficient in the case of the non-mineable group, indicating a lack of idiosyncratic volatility spillovers. As previously observed, both Currency and dApp cohorts shared this same volatility spillover characteristic, while idiosyncratic volatility spillovers were absent in the Protocol grouping. As we have previously identified, Protocols are more likely to be non-mineable, while Currencies are typical mined. These results are consistent with this early finding, and further confirm the increased likelihood of idiosyncratic volatility transfers in mineable assets, and a reduced propensity in Protocols.

Shift volatility spillovers is found to be both significant and positive, in both portfolios, further confirming the assertion that this form of volatility spillover is evenly distributed amongst all forms of asset, irrespective of category or mining status. As illustrated in table 6, both groups display evidence of volatility spillovers that were driven by US monetary policy announcements. Mineable assets are shown to be driven by multiple forms of volatility spillovers, while Non-Mineable assets are primarily volatility driven. The fact that neither grouping is driven by idiosyncratic volatility spillovers is surprising, when the previous section's results are considered. Multiple spillovers was found to be the driving form of volatility spillover for just $17 \%$ of individual assets. volatility-driven spillovers accounted for an even smaller figure of just $7 \%$. Additionally, idiosyncratic spillovers were found to be the primary driver of spillover effects in the Currency grouping.

\section{Robustness Testing}

Having previously subjected our analysis to a number of robustness checks based on groups denoted by both asset category and mining status - we further analyse our sample by sub-dividing it into a number of phases, and re-estimating the results. Such an approach has been used successfully in previous studies by Claessens et al. [2010]; Dungey and Gajurel [2015] and Corbet and Larkin [2017]. From our total sample of eight policy announcements (Table 2), we note that the first four were focused on a quantitative easing announcement or change, while the remaining four centred on an interest rate increase. The four $\mathrm{QE}$ announcements were made over a period of seventeen months spanning from 22 May 2013 to 29 October 2014, while the interest rate changes were made over a period of eighteen months, from 17 December 2015 to 15 June 2017. We divide our entire sample into two periods, based on these two date ranges, and label the QE announcement range Period I and the interest rate range Period II. The results are displayed in Table 7. The breaking of our entire sample provides an opportunity to differentiate between the early stages of the market, and the changes that have occurred as the market matures. Such a method is in line with the work of Bouoiyour and Selmi [2015] who note two distinct phases of BTC returns, and observe a maturing of the Bitcoin market in recent times.

\section{Insert Table 7 about here}

We incorporate these two periods into our existing model by extending equations (3) and (5) as follows, where $I_{1}$ and $I_{2}$ are indicator variables, taking a value 0 or 1 , relative to the appropriate period of our sample. 


$$
\begin{aligned}
r_{j, t}= & b_{0, j}+b_{1} f_{t}^{g l o b a l}+b_{2} f_{t}^{\text {global }} I_{1, t}+b_{3} f_{t}^{\text {global }} I_{2, t} \\
& +b_{4} f_{t}^{B T C}+b_{5} f_{t}^{B T C} I_{1, t}+b_{4} f_{t}^{B T C} I_{2, t} \\
& +b_{7} I_{1, t}+b_{8} I_{2, t}+\varepsilon_{i, t} \\
\ln \left(\sigma_{j, t}^{2}\right)= & c_{0, j}+c_{1, j}\left(\left|Z_{i, t-1}\right|-E \mid Z_{i, t-1}\right) \\
& +c_{2, j} z_{j, t-1}+c_{3, j} \ln \left(\sigma_{j, t-1}^{2}\right)+\pi_{1, j} \ln \left(\sigma_{B T C, t}^{2}\right) \\
& +\pi_{2, j} \ln \left(\sigma_{B T C, t}^{2}\right) I_{1, t}+\pi_{3, j} \ln \left(\sigma_{B T C, t}^{2}\right) I_{2, t}
\end{aligned}
$$

Results for periods I and II are presented in Table 5. With the exception of idiosyncratic, all other forms of volatility spillovers decreased to a lower level in Period 2. The second stage GARCH estimation indicates that the percentage exposure of assets to global systematic volatility spillovers decreased from $31.7 \%$ in Period 1 to $17.1 \%$ in Period 2, while the number of assets exposed to shift spillovers decreased from $46.3 \%$ to $31.7 \%$. This decrease in both systematic and shift spillovers suggests that digital assets become less exposed to such forms, as they mature. We observe an increase in market capitalisation across all digital assets, throughout our sample period (as illustrated in Figure 2), suggesting that this increase contributes to the reduction in systematic and shift volatility spillover exposure. Volatility spillovers exposure experienced a small decrease from $58.5 \%$ to $56 \%$. Volatility spillovers remains the most dominant and frequent form of volatility spillovers exposure in both Periods 1 and 2 . The relatively small change across periods suggests that levels of volatility spillovers exposure remain constant as asset size increases. Only idiosyncratic spillovers experience an increase between periods, with $39 \%$ of assets displaying evidence of exposure to such volatility spillovers in Period 2 representing an increase from $29.2 \%$ in Period 1 . This observed increase indicating that increases in the market capitalisation of digital assets correspond with an increase in levels of idiosyncratic volatility spillover exposure.

\section{Conclusions}

Our findings are consistent with our previous, theoretical, discussion on the existence of traditional volatility spillover channels between broad financial markets and the new digital asset class containing cryptocurrencies (Corbet et al. [2018]). Our primary observation is that digital assets do not in fact, react in an identical manner, and so, should not be viewed as one category or market. Currencies appear linked to the FIAT market, and remain connected (at least in a statistical sense), to the policy making decisions of traditional FIAT banks (Federal Reserve). While we isolated currencies from the rest of the application layer, we observe other forms of application to display a similar reaction. Our most significant finding however, is the observation that protocol based assets display evidence of a completely different reaction, in some cases moving in an opposite direction to currency based applications. Such findings confirm our previous proposition, and warrant digital assets to be evaluated based on their use, and place, within the blockchain stack rather than viewed as a single entity within the cryptocurrency market.

In the previously examined Internet stack, underlying web protocols were not accessible to investors, leaving only the top application layer as an avenue to create wealth. This model has changed with the advent of blockchain technology, and as a result, wealth will indeed be created in 
the lowest level of the stack. As such, these underlying protocols should be viewed as almost a new form of financial asset, one that cannot be compared with previous instruments, and our findings, the observation of their reaction to traditional FIAT markets and monetary policy announcements, confirm this to be the case. Application layer ICOs are being used as an alternative method to raise capital for projects, which in some cases are still at a concept stage, rather than utilising traditional venture capital funding routes. ICOs are being used to crowd-source funding, and in many cases, the process has been abused. The rapid growth of such ICOs present evidence of one of the traditional hallmarks of a bubble. If all assets and cryptocurrencies are viewed in this manner, it follows that the entire market would be subject to the effects of a bubble. Zeira [1999] offered an explanation for this through development of the hypothesis of 'informational overshooting', where bubbles are largely explained due to informational dynamics. This appears to be a explanation that is largely applicable to this rapidly developing market. As we have demonstrated, these blockchain applications are simply thin layer applications, built upon the protocols themselves. Once we differentiate between these two classes of digital assets, it becomes possible to consider these applications to be overvalued and in the midst of a bubble, whilst viewing protocols as entirely separate entities, indeed entities that could retain value despite a potential market crash that may occur among application layer tokens.

\section{Bibliography}

Akyildirim, E., S. Corbet, P. Katsiampa, N. Kellard, and A. Sensoy (2019). The development of bitcoin futures: Exploring the interactions between cryptocurrency derivatives. Finance Research Letters Article in Press.

Akyildirim, E., S. Corbet, B. Lucey, A. Sensoy, and L. Yarovaya (2019). The relationship between implied volatility and cryptocurrency returns. Finance Research Letters Article in Press.

Andersen, T. G. and T. Bollerslev (1997). Heterogeneous Information Arrivals and Return Volatility Dynamics: Uncovering the Long-Run in High Frequency Returns. The Journal of Finance 52(3), 975-1005.

Bariviera, A. F., M. J. Basgall, W. Hasperué, and M. Naiouf (2017). Some stylized facts of the Bitcoin market. Physica A: Statistical Mechanics and its Applications 484, 82-90.

Berger, A. and C. Bouwman (2017). Bank liquidity creation, monetary policy, and financial crises. Journal of Financial Stability 30, 139-155.

Böhme, R., N. Christin, B. Edelman, and T. Moore (2015). Bitcoin: Economics, Technology, and Governance. Journal of Economic Perspectives 29(2), 213-238.

Bouoiyour, J. and R. Selmi (2015). What does Bitcoin look like? Annals of Economics and Finance 16(2), 449-492.

Bouoiyour, J. and R. Selmi (2016). Bitcoin: A beginning of a new phase? Economics Bulletin 36(3), $1430-1440$.

Brandvold, M., P. Molnár, K. Vagstad, and O. C. Andreas Valstad (2015). Price discovery on Bitcoin exchanges. Journal of International Financial Markets, Institutions and Money 36, 18 35. 
Celeste, V., S. Corbet, and C. Gurdgiev (2019). Fractal dynamics and wavelet analysis: Deep volatility and return properties of bitcoin, ethereum and ripple. Quarterly Review of Economics and Finance.

Cheah, E. T. and J. Fry (2015). Speculative bubbles in Bitcoin markets? An empirical investigation into the fundamental value of Bitcoin. Economics Letters 130, 32-36.

Cheung, A. W. K., E. Roca, and J. J. Su (2015). Crypto-currency bubbles: an application of the Phillips, Shi and Yu (2013) methodology on Mt. Gox bitcoin prices. Applied Economics 47(23), $2348-2358$.

Christopoulos, A. and R. Jarrow (2018). Cmbs market efficiency: The crisis and the recovery. Journal of Financial Stability 36, 159-186.

Claessens, S., G. Dell'Ariccia, D. Igan, and L. Laeven (2010). Cross-country experiences and policy implications from the global financial crisis. Economic Policy 25(62), 267-293.

Corbet, S., V. Eraslan, B. M. Lucey, and A. Sensoy (2019). The effectiveness of technical trading rules in cryptocurrency markets. Finance Research Letters 31, 32-37.

Corbet, S. and C. Larkin (2017). Has the uniformity of banking regulation within the European Union restricted rather than encouraged sectoral development? International Review of Financial Analysis 53, 48-65.

Corbet, S., B. Lucey, A. Urquhart, and L. Yarovaya (2019). Cryptocurrencies as a financial asset: A systematic analysis. International Review of Financial Analysis 62, 182-199.

Corbet, S., B. Lucey, and L. Yarovaya (2018). Datestamping the bitcoin and ethereum bubbles. Finance Research Letters 26, 81-88.

Corbet, S., A. Meegan, C. Larkin, B. Lucey, and L. Yarovaya (2018). Exploring the dynamic relationships between cryptocurrencies and other financial assets. Economics Letters 165, 28-34.

Dungey, M. and D. Gajurel (2015). Contagion and banking crisis - International evidence for 2007-2009. Journal of Banking and Finance 60, 271-283.

Dwyer, G. P. (2015). The economics of Bitcoin and similar private digital currencies. Journal of Financial Stability 17, 81-91.

Edwards, S. (1998). Interest Rate Volatility, Capital Controls, and Contagion. National Bureau of Economic Research Working Paper Series No. 6756.

Eken, M. H. and E. Baloglu (2017). Crypto currencies and their destinies in the future. Journal of Finance 83 Banking Studies 6(4), 1-11.

Engle, R. F., T. Ito, W. L. Lin, and W. L. Lin (1990). Meteor showers or heat waves? Heteroskedastic intra-daily volatility in the foreign exchange market. Econometrica 58(3), 525-542.

Forbes, K. J. and R. Rigobon (2002). No Contagion, Only Interdependence: Measuring Stock Market Comovements. The Journal of Finance 57(5), 2223-2261. 
Fry, J. (2018). Booms, busts and heavy-tails: The story of bitcoin and cryptocurrency markets? Economics Letters 171, 225-229.

Fry, J. and E. T. Cheah (2016). Negative bubbles and shocks in cryptocurrency markets. International Review of Financial Analysis 47, 343-352.

Gandal, N., J. Hamrick, T. Moore, and T. Oberman (2018). Price manipulation in the Bitcoin ecosystem. Journal of Monetary Economics 95, 86-96.

Gertler, P. and R. Horvath (2018). Central bank communication and financial markets: New high-frequency evidence. Journal of Financial Stability 36, 336-345.

Gronwald, M. (2014). The economics of Bitcoin: Market characteristics and price jumps. CESifo Working Paper (5121).

Katsiampa, P., S. Corbet, and B. Lucey (2019a). High frequency volatility co-movements in cryptocurrency markets. Journal of International Financial Markets, Institutions and Money 62, $35-52$.

Katsiampa, P., S. Corbet, and B. Lucey (2019b). Volatility spillover effects in leading cryptocurrencies: A BEKK-MGARCH analysis. Finance Research Letters, 29, 68-74.

Krainer, R. (2017). Economic stability under alternative banking systems: Theory and policy. Journal of Financial Stability 31, 107-118.

Kroll, J., I. Davey, and E. Felten (2013). The Economics of Bitcoin Mining, or Bitcoin in the Presence of Adversaries. The Twelfth Workshop on the Economics of Information Security (WEIS 2013) (WEIS), 1-21.

Masson, P. (1999). Contagion: macroeconomic models with multiple equilibria. Journal of International Money and Finance 18, 587-602.

Mester, L. (2017). The nexus of macroprudential supervision, monetary policy, and financial stability. Journal of Financial Stability 30, 177-180.

Nadarajah, S. and J. Chu (2017). On the inefficiency of Bitcoin. Economics Letters 150, 6-9.

Nakamoto, S. (2008). Bitcoin: A Peer-to-Peer Electronic Cash System. Available at: www.bitcoin.org, 9 .

Phillips, P. C., S. Shi, and J. Yu (2015). Testing for multiple bubbles: Historical episodes of exuberance and collapse in the S\&P 500. International Economic Review 56(4), 1043-1078.

Risteski, D. and D. Davcev (2014). Can we use daily internet search query data to improve predicting power of egarch models for financial time series volatility. In Proceedings of the International Conference on Computer Science and Information Systems (ICSIS-2014), October 17-18, 2014, Dubai (United Arab Emirates).

Sensoy, A. (2019). The inefficiency of Bitcoin revisited: A high-frequency analysis with alternative currencies. Finance Research Letters, 28, 68-73.

Urquhart, A. (2017). Price clustering in Bitcoin. Economics Letters 159, 145-148. 
Yoldas, E. and Z. Senyuz (2018). Financial stress and equilibrium dynamics in term interbank funding markets. Journal of Financial Stability 34, 136-149.

Zeira, J. (1999). Informational overshooting, booms, and crashes. Journal of Monetary Economics 43(1), 237-257. 
Figure 1: An illustration of the TCP/IP internet stack and suggested blockchain stack

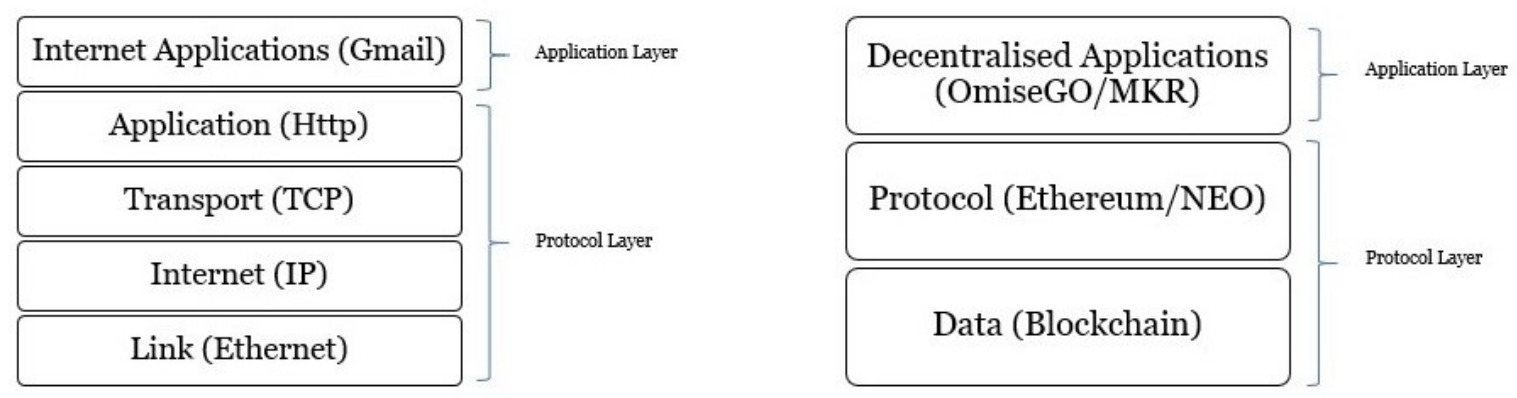

Note: The above figure contains a number of examples of internet protocols including: Hypertext Transfer Protocol (HTTP), the underlying protocol of the World Wide Web defining how messages are formatted and transmitted, HTTP Secure (HTTPS), a communications protocol used for secure communication over networks, and Secure Sockets Layer (SSL), a communications security protocol commonly used in email. Unlike Currencies, protocols are digital assets whose primary function will be that of data transfer, and provide a bottom layer on which to build decentralised applications. When categorising the digital assets in our sample, we include any platform, blockchain or method for data transfer, upon which other applications could feasibly be built, in our definition of the term "protocol". Decentralised Applications (dApps) refers to any application whose back-end code runs on a decentralised peer-to-peer network. This contrasts with traditional applications, whose back-end code is usually executed on a centralised server. dApps typically consist of front-end code and user interfaces written in a traditional programming language, and back-end code executed on a blockchain - typically in the form of smart contracts. 
Table 1: Sample of digital assets included in this analysis

\begin{tabular}{|c|c|c|c|c|c|}
\hline Ticker & Name & Ticker & Name & Ticker & Name \\
\hline$\overline{\mathrm{BTC}}$ & Bitcoin & DCR & Decred & PPY & Peerplays \\
\hline ETH & Ethereum & KMD & Komodo & $\mathrm{LBC}$ & LBRY Credits \\
\hline $\mathrm{XRP}$ & Ripple & DGB & DigiByte & NMC & Namecoin \\
\hline LTC & Litecoin & DGD & DigixDAO & QRL & $\begin{array}{l}\text { Quantum Re- } \\
\text { sistant Ledger }\end{array}$ \\
\hline ETC & Ethereum Classic & NXT & Nxt & WINGS & Wings \\
\hline XEM & NEM & BAT & Basic Attention Token & AMP & Synereo \\
\hline DASH & Dash & PIVX & PIVX & BAY & BitBay \\
\hline MIOTA & IOTA & $1 \mathrm{ST}$ & FirstBlood & STORJ & Storj \\
\hline BTS & BitShares & $\mathrm{BNT}$ & Bancor & MONA & MonaCoin \\
\hline XMR & Monero & SNGLS & SingularDTV & CLOAK & CloakCoin \\
\hline STRAT & Stratis & MGO & MobileGo & VSL & vSlice \\
\hline EOS & EOS & MCAP & MCAP & $\mathrm{XCP}$ & Counterparty \\
\hline $\mathrm{ZEC}$ & Zcash & BTCD & BitcoinDark & XEL & Elastic \\
\hline WAVES & Waves & SYS & SysCoin & BLK & BlackCoin \\
\hline STEEM & Steem & FUN & FunFair & NLG & Gulden \\
\hline ANS & AntShares & ANT & Aragon & OBITS & OBITS \\
\hline $\mathrm{BCN}$ & Bytecoin & NXS & Nexus & XAUR & Xaurum \\
\hline GNT & Golem & XAS & Asch & VIA & Viacoin \\
\hline VERI & Veritaseum & UBQ & Ubiq & OMNI & Omni \\
\hline $\mathrm{SC}$ & Siacoin & LKK & Lykke & $\mathrm{XZC}$ & Zcoin \\
\hline $\mathrm{BCC}$ & BitConnect & PPC & Peercoin & BURST & Burst \\
\hline GNO & Gnosis & EMC & Emercoin & HMQ & Humaniq \\
\hline $\mathrm{ICN}$ & Iconomi & ARK & Ark & VTC & Vertcoin \\
\hline REP & Augur & ROUND & Round & SLS & Salus \\
\hline LSK & Lisk & EDG & Edgeless & MYST & Mysterium \\
\hline XLM & Stellar Lumens & LEO & LEOcoin & BLOCK & Blocknet \\
\hline DOGE & Dogecoin & RDD & ReddCoin X & AGRS & Agoras Tokens \\
\hline GBYTE & Byteball & SJCX & Storjcoin & YBC & YbCoin \\
\hline MAID & MaidSafeCoin & NMR & Numeraire & $\mathrm{EAC}$ & EarthCoin \\
\hline GAME & GameCredits & DICE & Etheroll & NAV & NAV Coin \\
\hline $\mathrm{FCT}$ & Factom & RLC & iExec RLC & GRC & GridCoin \\
\hline USDT & Tether & XVG & Verge & TKN & TokenCard \\
\hline ARDR & Ardor & MLN & Melon & $\mathrm{QAU}$ & Quantum \\
\hline SNT & Status & & & & \\
\hline
\end{tabular}

Note: Digital Assets sample consisting of Top 100 digital assets by market capitalisation on June 15th, 2017. Only assets with more than 90 daily observations were included in the final sample. Data sourced from www.coinmarketcap.com. 
Figure 2: GARCH-calculated volatility distributed by market capitalisation and type of cryptocurrency
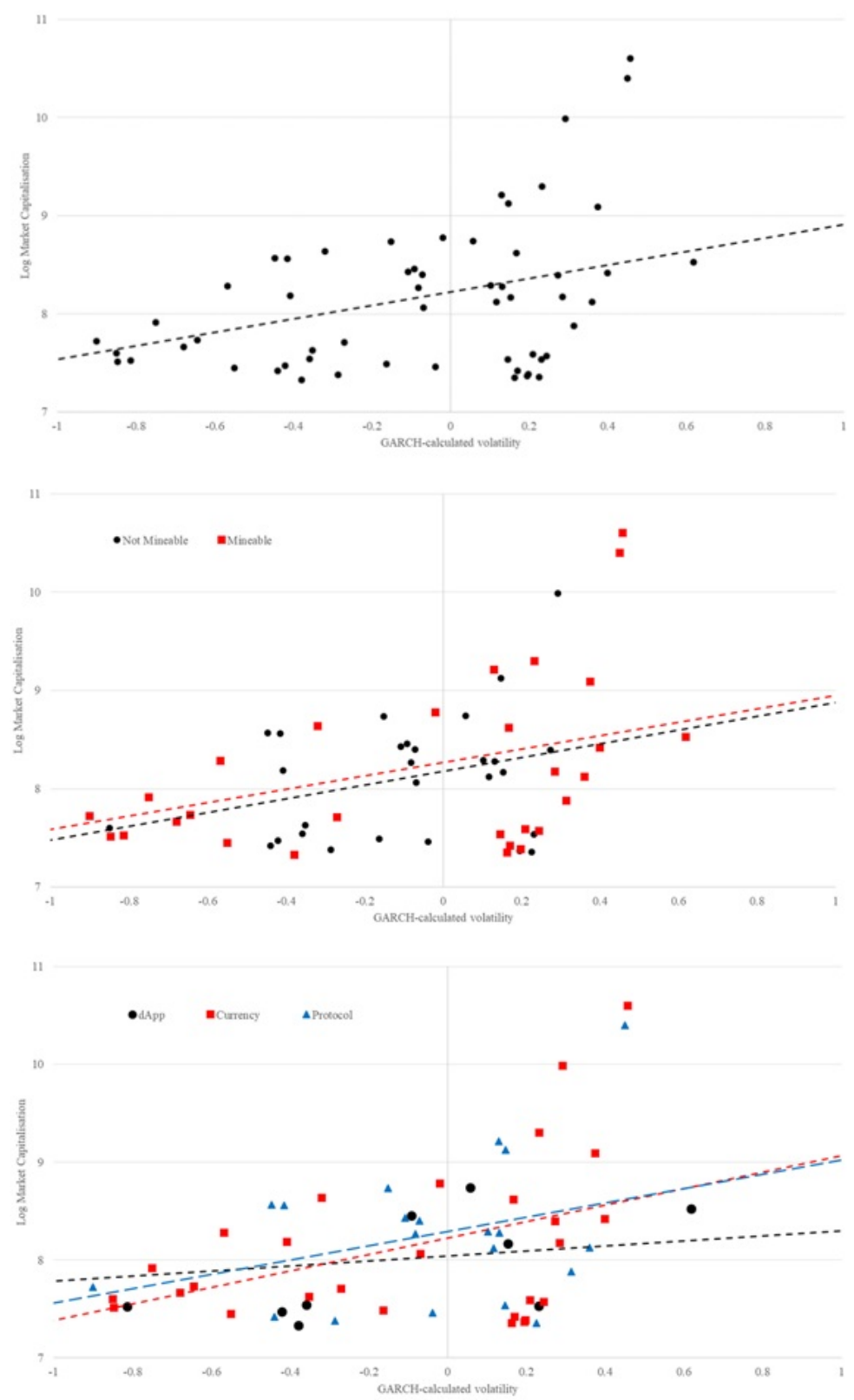

Note: The top figure represents the entire sample of cryptocurrencies. The centre figure divides the sample into those cryptocurrencies denoted as mineable and non-mineable. The bottom figure divides the sample into those cryptocurrencies denotes as a currency, a protocol or a dApp. The vertical axes in all three figures represents the log of the crytocurrency's market capitalisation, whereas the horizontal axes represents the GARCH-calculated volatility. 
Table 2: FOMC Policy Announcements

\begin{tabular}{llcc}
\hline \hline Date & Type & Change & New Level \\
\hline & & & \\
15 June 2017 & Fed Fund Rate & $+0.25 \%$ & $1.00 \%$ to $1.25 \%$ \\
16 March 2017 & Fed Fund Rate & $+0.25 \%$ & $0.75 \%-1.00 \%$ \\
15 December 2016 & Fed Fund Rate & $+0.25 \%$ & $0.50 \%-0.75 \%$ \\
17 December 2015 & Fed Fund Rate & $+0.25 \%$ & $0.25 \%-0.50 \%$ \\
29 October 2014 & QE3 End & End & $0 \%$ \\
18 December 2013 & QE3 Taper & $-\$ 10$ Billion/month & $\$ 80$ Billion/Month \\
19 June 2013 & QE (May Scale Back Event) & - & - \\
22 May 2013 & QE (Taper Tantrum) & - & - \\
\hline
\end{tabular}

Note: FOMC Federal Fund target rate and QE policy announcements from 2013 - 2017 
Table 3: GARCH Methodology Selection Criteria

\begin{tabular}{|c|c|c|c|c|c|c|c|c|c|c|}
\hline Model & \multicolumn{2}{|c|}{ GARCH } & \multicolumn{2}{|c|}{ EGARCH } & \multicolumn{2}{|c|}{ TGARCH } & \multicolumn{2}{|c|}{ GJR-GARCH } & \multicolumn{2}{|c|}{ Best Identified Methodology } \\
\hline Crypto & AIC & BIC & AIC & $\mathrm{BIC}$ & AIC & BIC & AIC & $\mathrm{BIC}$ & AIC & BIC \\
\hline$\overline{\text { AGRS }}$ & -327.878 & -312.984 & -367.418 & -348.801 & -358.285 & -339.667 & -353.145 & -334.528 & EGARCH & EGARCH \\
\hline ANS & -184.180 & -170.986 & -211.898 & -195.196 & -205.945 & -189.453 & -204.137 & -187.645 & EGARCH & EGARCH \\
\hline BAY & -884.417 & -862.017 & -914.274 & -905.314 & -895.572 & -868.692 & -887.833 & -860.952 & EGARCH & EGARCH \\
\hline $\mathrm{nBCN}$ & -698.131 & -674.984 & -773.729 & -755.769 & -772.859 & -745.083 & -749.609 & -721.833 & EGARCH & EGARCH \\
\hline BLOCK & -373.892 & -351.423 & -376.977 & -350.015 & -374.568 & -347.605 & -277.386 & -259.609 & EGARCH & GARCH \\
\hline BTC & $-3,597.725$ & $-3,577.945$ & $-3,687.721$ & $-3,682.776$ & $-3,600.457$ & $-3,575.731$ & $-3,608.627$ & $-3,583.902$ & EGARCH & EGARCH \\
\hline $\mathrm{BTCC}$ & -128.260 & -109.850 & -442.153 & -419.140 & -243.205 & -220.192 & -242.834 & -224.601 & EGARCH & EGARCH \\
\hline BTS & $-1,694.991$ & $-1,671.999$ & $-1,790.792$ & $-1,723.201$ & $-1,748.595$ & $-1,721.004$ & $-1,783.014$ & $-1,755.423$ & EGARCH & GJR-GARCH \\
\hline BURST & -938.483 & -915.699 & $-1,062.014$ & $-1,052.901$ & -984.423 & -957.083 & -999.782 & -972.442 & EGARCH & EGARCH \\
\hline CLOAK & -25.700 & -2.514 & -103.814 & -94.540 & 4.474 & 32.298 & -53.973 & -26.150 & EGARCH & EGARCH \\
\hline DASH & $-1,858.894$ & $-1,835.215$ & $-1,876.893$ & $-1,848.478$ & $-1,861.448$ & $-1,833.033$ & $-1,870.142$ & $-1,841.727$ & EGARCH & EGARCH \\
\hline $\mathrm{DCR}$ & -585.422 & -566.161 & -588.274 & -565.161 & -584.196 & -561.083 & -587.028 & -565.915 & EGARCH & GARCH \\
\hline DGB & -939.185 & -915.470 & -988.410 & -978.924 & -688.779 & -660.322 & -983.925 & $-1,005.467$ & EGARCH & GJR-GARCH \\
\hline DGD & -597.759 & -579.223 & -597.838 & -580.424 & -551.139 & -528.896 & -594.911 & -572.668 & EGARCH & EGARCH \\
\hline DOGE & $-2,334.148$ & $-2,310.237$ & $-2,346.076$ & $-2,317.383$ & $-2,341.622$ & $-2,312.929$ & $-2,227.610$ & $-2,208.629$ & EGARCH & EGARCH \\
\hline EAC & $-1,020.506$ & -996.646 & $-1,214.286$ & $-1,204.742$ & $-1,028.639$ & $-1,000.007$ & $-1,044.429$ & $-1,015.797$ & EGARCH & EGARCH \\
\hline EMC & -649.228 & -627.743 & -688.824 & -680.230 & -657.328 & -631.545 & -678.849 & -653.067 & EGARCH & EGARCH \\
\hline ETC & -329.801 & -312.546 & -296.626 & -275.920 & -378.996 & -358.290 & -384.465 & -363.759 & GJR-GARCH & GJR-GARCH \\
\hline ЕTH & -871.243 & -850.448 & -909.305 & -884.350 & -877.852 & -852.897 & -908.754 & -883.799 & EGARCH & EGARCH \\
\hline $\mathrm{FCT}$ & -535.104 & -514.762 & -407.238 & -382.827 & -79.926 & -71.790 & -581.111 & -556.701 & GJR-GARCH & GJR-GARCH \\
\hline GAME & -468.673 & -446.413 & -477.974 & -451.262 & -470.109 & -443.396 & -406.594 & -388.993 & EGARCH & EGARCH \\
\hline ICN & -186.043 & -169.942 & -179.888 & -160.566 & -184.121 & -164.799 & -162.315 & -150.193 & GARCH & GARCH \\
\hline LBC & -144.019 & -126.513 & -162.117 & -141.109 & -147.549 & -126.541 & -150.057 & -129.049 & EGARCH & EGARCH \\
\hline LEO & -717.917 & -697.090 & -721.559 & -696.567 & -650.325 & -633.942 & -719.892 & -694.899 & EGARCH & GARCH \\
\hline LSK & -534.411 & -515.745 & -529.384 & -506.984 & -532.894 & -510.494 & -530.803 & -508.403 & GARCH & GARCH \\
\hline LTC & $-3,402.561$ & $-3,377.835$ & $-3,604.928$ & $-3,595.038$ & $-3,410.770$ & $-3,381.100$ & $-3,423.087$ & $-3,393.417$ & EGARCH & EGARCH \\
\hline MAID & $-1,799.733$ & $-1,776.361$ & $-1,806.999$ & $-1,778.952$ & $-1,801.393$ & $-1,773.346$ & $-1,798.922$ & $-1,770.875$ & EGARCH & EGARCH \\
\hline MONA & $-1,633.403$ & $-1,605.861$ & $-1,638.937$ & $-1,615.985$ & $-1,583.449$ & $-1,555.907$ & $-1,632.188$ & $-1,604.646$ & EGARCH & EGARCH \\
\hline NLG & $-1,275.923$ & $-1,252.462$ & $-1,374.767$ & $-1,365.382$ & $-1,280.800$ & $-1,252.647$ & $-1,318.722$ & $-1,290.569$ & EGARCH & EGARCH \\
\hline NMC & $-2,698.968$ & $-2,674.243$ & $-2,759.274$ & $-2,749.384$ & $-2,697.461$ & $-2,667.790$ & $-2,725.263$ & $-2,695.592$ & & EGARCH \\
\hline NXS & -529.152 & -507.648 & -540.762 & -514.958 & -529.177 & -503.372 & -534.560 & -508.756 & EGARCH & EGARCH \\
\hline $\mathrm{NXT}$ & $-1,697.809$ & $-1,673.853$ & $-1,832.077$ & $-1,822.495$ & $-1,762.519$ & $-1,733.771$ & $-2,180.471$ & $-2,161.452$ & GJR-GARCH & GJR-GARCH \\
\hline PIVX & -145.910 & -126.692 & -211.764 & -204.077 & -139.364 & -116.303 & -178.295 & -155.234 & EGARCH & EGARCH \\
\hline PPC & $-2,671.355$ & $-2,647.010$ & $-2,686.806$ & $-2,657.592$ & $-2,684.418$ & $-2,655.204$ & $-2,493.824$ & $-2,474.483$ & EGARCH & EGARCH \\
\hline RDD & -448.755 & -425.124 & -458.471 & -430.114 & -485.698 & -457.341 & -460.028 & -431.671 & TGARCH & TGARCH \\
\hline REP & -371.617 & -354.810 & -375.442 & -368.720 & -331.324 & -311.156 & -366.518 & -346.350 & EGARCH & EGARCH \\
\hline $\mathrm{SC}$ & -540.398 & -520.776 & -560.006 & -536.461 & -479.239 & -471.390 & -564.307 & -540.762 & GJR-GARCH & GJR-GARCH \\
\hline SJCX & $-1,021.397$ & -998.577 & $-1,136.430$ & $-1,109.047$ & $-1,039.853$ & $-1,012.469$ & $-1,083.176$ & $-1,065.106$ & EGARCH & EGARCH \\
\hline $\mathrm{ST}$ & -249.799 & -240.669 & -289.562 & -283.100 & -270.407 & -251.020 & -267.324 & -247.937 & EGARCH & EGARCH \\
\hline STEEM & -133.665 & -115.130 & -177.852 & -160.438 & -160.472 & -138.229 & -176.179 & -153.936 & EGARCH & EGARCH \\
\hline SYS & -846.794 & -823.954 & -857.537 & -830.128 & -871.311 & -843.903 & -720.243 & -702.154 & TGARCH & TGARCH \\
\hline VIA & -749.818 & -726.819 & -767.519 & -739.920 & -756.064 & -728.465 & $-1,093.856$ & $-1,075.634$ & GJR-GARCH & GJR-GARCH \\
\hline VTC & $-1,319.072$ & $-1,295.287$ & $-1,319.163$ & $-1,309.649$ & -929.248 & -900.707 & $-1,261.339$ & $-1,242.463$ & EGARCH & EGARCH \\
\hline WAVES & -497.608 & -479.634 & -498.465 & -476.897 & -496.525 & -474.957 & -490.515 & -468.946 & EGARCH & $\mathrm{GARCH}$ \\
\hline XAUR & -567.987 & -546.446 & -585.792 & -577.176 & -566.110 & -540.261 & -520.192 & -494.344 & EGARCH & EGARCH \\
\hline $\mathrm{XCP}$ & $-1,407.215$ & $-1,383.566$ & $-1,435.214$ & $-1,406.835$ & $-1,432.043$ & $-1,403.664$ & $-1,431.282$ & $-1,402.903$ & EGARCH & EGARCH \\
\hline XEM & -724.322 & -702.655 & -823.300 & -797.300 & -726.151 & -700.152 & -799.712 & -773.713 & EGARCH & EGARCH \\
\hline XLM & $-1,644.687$ & $-1,621.770$ & $-1,745.599$ & $-1,718.099$ & $-1,679.719$ & $-1,652.219$ & $-1,708.615$ & $-1,681.115$ & EGARCH & EGARCH \\
\hline XMR & $-1,609.105$ & $-1,585.910$ & $-1,681.937$ & $-1,656.105$ & $-1,454.409$ & $-1,426.577$ & $-1,683.441$ & $-1,655.610$ & GJR-GARCH & EGARCH \\
\hline XRP & $-2,554.636$ & $-2,530.239$ & $-2,698.560$ & $-2,688.802$ & $-2,556.010$ & $-2,526.734$ & $\begin{array}{l}-2,539.130 \\
\end{array}$ & $-2,509.854$ & EGARCH & EGARCH \\
\hline$X V G$ & 269.130 & 282.486 & 132.771 & 159.779 & 401.974 & 428.982 & 212.235 & 239.242 & EGARCH & EGARCH \\
\hline YBC & $-2,364.157$ & $-2,340.360$ & $-2,447.559$ & $-2,419.003$ & $-2,370.580$ & $-2,342.025$ & $-2,382.302$ & $-2,353.747$ & EGARCH & EGARCH \\
\hline $\mathrm{ZEC}$ & -218.057 & -202.528 & -298.075 & -279.440 & -202.928 & -184.293 & -228.292 & -209.656 & EGARCH & EGARCH \\
\hline
\end{tabular}

Note: We considered the use of GARCH, Threshold GARCH (TGARCH) and GJR-GARCH, but EGARCH was found to outperform each methodology. Results are available from the authors on request. An intercept and a deterministic trend were included in the Augmented Dickey Fuller (ADF) and Phillips Perron (PP) models. The trend was included to capture the reduction in average volatility that took place during the period prior to the inclusion of CFDs. The ADF model tests whether the equity series contain a unit root in order to correct for serial correlation. PP tests employ a non-parametric estimator of the variance-covariance matrix with d truncation lags. The models test down by sequentially removing the last lag until a significant lag is reached giving the order of augmentation for the ADF test that minimised the Akaike information criterion. The results indicated rejected of the null-unit root hypotheses at a minimum of the 5 per cent level. Where necessary, we display the results of the best-fitting methodology as determined by the selection criteria. Further results for all methodological calculations are available from the authors on request. 
Table 4: Parameter results based on cryptocurrency volatility spillover effects before the investigated monetary policy announcements

\begin{tabular}{|c|c|c|c|c|c|c|c|c|c|c|}
\hline Asset & Category & Mineable & $b^{2}$ & $b^{4}$ & $b^{5}$ & $\pi^{2}$ & $b^{2}=b^{4}=0$ & $b^{2}=\pi^{2}=0$ & $b^{4}=\pi^{2}=0$ & $b^{2}=b^{4}=\pi^{2}=0$ \\
\hline \multicolumn{11}{|c|}{ Panel A: Idiosyncratic Volatility Spillovers } \\
\hline BTC & Currency & Yes & -0.755 & $0.505^{* * *}$ & $0.000^{* * *}$ & 0.507 & $30.59 * * *$ & 3.74 & $72.83^{* * *}$ & $68.51 * * *$ \\
\hline DASH & Currency & Yes & -0.177 & $0.098^{* * *}$ & $0.003^{*}$ & 0.416 & $14.82^{* * *}$ & 2.06 & $56.90^{* * *}$ & $68.89^{* * *}$ \\
\hline DCR & Currency & Yes & -0.277 & $0.089^{* * *}$ & $0.004^{* *}$ & $0.316^{* * *}$ & $5.13^{* *}$ & 0.42 & $3.01 *$ & $4.27 * *$ \\
\hline DOGE & Currency & Yes & 0.128 & $0.087^{* * *}$ & $-0.004^{* * *}$ & $0.444^{*}$ & $4.56^{* * *}$ & 2.32 & $7.54^{* * *}$ & $7.78^{* * *}$ \\
\hline EMC & Protocol & Yes & -0.371 & $0.706^{* * *}$ & $0.001^{* * *}$ & -1.012 & $3.27^{*}$ & 1.10 & $5.94^{* * *}$ & $6.30^{* * *}$ \\
\hline ICN & dApp & No & -0.107 & $0.831^{* *}$ & $-0.001 * *$ & $-0.091^{* *}$ & $4.94^{* *}$ & 1.70 & $8.94^{* * *}$ & $6.63^{* * *}$ \\
\hline $\mathrm{LBC}$ & dApp & Yes & 0.829 & $0.192^{* * *}$ & $0.030^{* * *}$ & $0.813^{* *}$ & $3.09^{* *}$ & 0.01 & $4.59^{* *}$ & $7.99^{* *}$ \\
\hline LTC & Currency & Yes & -0.149 & $0.122^{* * *}$ & $-0.001 *$ & -0.258 & $7.29^{* * *}$ & 1.12 & $21.52^{* * *}$ & $26.43^{* * *}$ \\
\hline NXS & Currency & Yes & -0.581 & $0.563^{* *}$ & $-0.001^{* * *}$ & $-0.754 * * *$ & $3.49^{* *}$ & 1.55 & $5.97^{* * *}$ & $10.05^{* * *}$ \\
\hline PPC & Currency & Yes & $-0.239^{*}$ & $1.013^{* * *}$ & $0.003^{* * *}$ & -0.714 & $7.17^{* * *}$ & 1.73 & $10.81^{* * *}$ & $12.54^{* * *}$ \\
\hline \multicolumn{11}{|c|}{ Panel B: Multiple Drivers of Volatility Spillovers } \\
\hline ANS & Protocol & No & $-0.124^{* * *}$ & 0.149 & 0.002 & $-0.495^{*}$ & $24.47 * * *$ & $21.55^{* * *}$ & $12.78^{* * *}$ & $43.88^{* * *}$ \\
\hline $\mathrm{BCN}$ & Currency & Yes & $0.107^{* *}$ & $-0.054^{* *}$ & $-0.004^{* *}$ & $0.186^{* * *}$ & $5.48^{* * *}$ & $4.62^{* * *}$ & $12.43^{* * *}$ & $30.22 * * *$ \\
\hline BTCD & Currency & Yes & $0.102^{* * *}$ & $0.286^{* * *}$ & $-0.003^{* * *}$ & $0.832^{* * *}$ & $10.66^{* * *}$ & $17.68^{* * *}$ & $13.91^{* * *}$ & $21.38^{* * *}$ \\
\hline BURST & Currency & Yes & $0.115^{* * *}$ & $1.063^{* * *}$ & $0.004^{* * *}$ & $0.181 *$ & $18.21 * * *$ & $20.55^{* * *}$ & $34.34^{* * *}$ & $41.89 * * *$ \\
\hline ETH & Protocol & Yes & $-0.606^{* * *}$ & $-0.323^{* *}$ & $0.003^{* * *}$ & $0.491^{* *}$ & $7.98^{* * *}$ & $10.61^{* * *}$ & $45.18^{* * *}$ & $60.78^{* * *}$ \\
\hline LEO & Currency & Yes & $1.054^{* * *}$ & $0.163^{* *}$ & $-0.002^{* * *}$ & $-0.298^{* * *}$ & $42.75^{* * *}$ & $45.95^{* * *}$ & $53.48^{* * *}$ & $127.25^{* * *}$ \\
\hline MONA & Currency & Yes & $0.615^{* * *}$ & 0.563 & $0.001^{* * *}$ & 0.233 & $15.07 * * *$ & $17.04^{* * *}$ & $41.09 * * *$ & $68.46^{* * *}$ \\
\hline NXT & Protocol & No & $-0.046^{* * *}$ & $0.094 * * *$ & $0.001 *$ & $0.129^{* * *}$ & $11.14^{* * *}$ & $8.46^{* * *}$ & $17.27^{* * *}$ & $26.63^{* * *}$ \\
\hline STRAT & Protocol & No & $-0.144^{* * *}$ & $0.127^{* * *}$ & -0.001 & $-0.167^{*}$ & $9.06^{* * *}$ & $7.35^{* *}$ & $22.45^{* * *}$ & $34.53^{* * *}$ \\
\hline ZEC & Currency & Yes & 0.129 & $0.052^{* * *}$ & 0.001 & $-0.354^{* * *}$ & $3.22^{*}$ & $3.22^{* *}$ & $3.32^{* *}$ & $3.65^{* * *}$ \\
\hline \multicolumn{11}{|c|}{ Panel C: Systematic Volatility Spillovers } \\
\hline AGRS & Protocol & No & -0.353 & $0.913 * * *$ & 0.004 & $0.251 *$ & $3.37^{* *}$ & $4.22^{*}$ & 0.83 & $5.44^{*}$ \\
\hline NLG & Currency & Yes & -0.272 & $0.916^{* * *}$ & 0.006 & -0.941 & $9.23^{* * *}$ & $15.26^{* * *}$ & 0.49 & $15.76^{* * *}$ \\
\hline RDD & Currency & No & $2.053^{* * *}$ & -0.238 & -0.001 & 0.389 & $30.62^{* * *}$ & $27.02 * * *$ & 0.74 & $71.58^{* * *}$ \\
\hline XAUR & Currency & No & -0.408 & $0.174^{* * *}$ & -0.003 & -0.181 & $4.33^{*}$ & $33.26^{* * *}$ & 0.84 & $39.17^{* * *}$ \\
\hline XLM & Currency & No & $0.289^{* *}$ & $0.045^{* * *}$ & 0.003 & $0.303^{*}$ & $4.16^{*}$ & $4.77^{* *}$ & 1.80 & $7.06^{* *}$ \\
\hline \multicolumn{11}{|c|}{ Panel D: Volatility Driven Spillovers } \\
\hline CLOAK & Currency & No & 0.533 & 0.471 & -0.004 & $-0.943^{* * *}$ & 0.43 & $7.23 * *$ & $13.14^{* * *}$ & $14.06^{* * *}$ \\
\hline $\mathrm{EAC}$ & Currency & Yes & 0.325 & $0.883^{* * *}$ & $-0.002^{* *}$ & $0.181^{* * *}$ & 1.24 & $4.31 * *$ & $54.98 * * *$ & $56.31 * * *$ \\
\hline SYS & Protocol & Yes & 0.261 & $0.915^{* * *}$ & $0.001^{*}$ & $0.348^{* *}$ & 0.24 & $4.29 * *$ & $7.88^{* * *}$ & $7.95^{* *}$ \\
\hline XEM & Protocol & No & 0.834 & 0.105 & 0.004 & $0.164^{* * *}$ & 1.59 & $3.14^{* *}$ & $3.18^{*}$ & $5.21^{*}$ \\
\hline
\end{tabular}

Note: The values for $b_{2}, b_{3}, b_{4}, b_{5}$ and $\pi_{2}$ are the parameter estimates and values for joint test are the Chi-square values. For brevity, we have not presented the results of methodologies that did not present significant results. These results are available on request. Tests of $\pi^{2}(20)$ and $\pi^{2}(30)$ were used as a robustness check for the decision to avail of a 50-day dummy variable window. ${ }^{* * *}$, ${ }^{* *}$, and $*$ indicate level of significance at $1 \%, 5 \%$, and $10 \%$ respectively. 
Table 5: Grouping Parameter Results: Currency, Protocol, dApp

\begin{tabular}{lcccccccccc}
\hline Asset & $b^{2}$ & $b^{4}$ & $b^{5}$ & $\pi^{2}$ & $b^{2}=b^{4}=0$ & $b^{2}=\pi^{2}=0$ & $b^{4}=\pi^{2}=0$ & $b^{2}=b^{4}=\pi^{2}=0$ & Spillover Type \\
Currency & -0.298 & $0.176^{* * *}$ & $0.001^{* * *}$ & $1.046^{* * *}$ & $9.83^{* * *}$ & 2.12 & $7.15^{* * *}$ & $7.49^{* * *}$ & Idiosyncratic \\
dApp & -0.217 & $0.307^{* * *}$ & $0.001^{* * *}$ & $-0.397^{* * *}$ & 0.54 & 0.61 & 1.19 & 1.63 \\
Protocol & -0.280 & 0.137 & $0.001^{* *}$ & $0.062^{*}$ & 0.84 & 0.79 & 0.32 & 1.31 & None \\
\hline
\end{tabular}

Note: The above table presents the parameter estimates indicating the effects of US policy announcements on 58 digital assets. The sample is sorted into thre cohorts, Currency, Protocol and dApp, based on the descriptions in Section 3. Coefficients $b^{2}, b^{4}$ and $b^{5}$ represent additional global systematic risk exposure during the announcement period (systematic volatility spillovers), additional US shocks during announcement period (idiosyncratic volatility spillovers) an intercept shift (shift volatility spillovers), respectively. $\pi^{2}$ represents additional US volatility spillovers during the announcement period (volatility driven spillovers). $* * *, * *$, and $*$ indicate level of significance at $1 \%, 5 \%$, and $10 \%$ respectively.

Table 6: Grouping Parameter Results: Mineable, Non-Mineable

\begin{tabular}{|c|c|c|c|c|c|c|c|c|c|}
\hline Asset & $b^{2}$ & $b^{4}$ & $b^{5}$ & $\pi^{2}$ & $b^{2}=b^{4}=0$ & $b^{2}=\pi^{2}=0$ & $b^{4}=\pi^{2}=0$ & $b^{2}=b^{4}=\pi^{2}=0$ & Spillover Type \\
\hline Yes & -1.087 & $0.114^{* *}$ & $0.000 * * *$ & $1.956^{* * *}$ & $14.46^{* * *}$ & $39.00^{* * *}$ & $62.99^{* * *}$ & $63.12^{* * *}$ & Multiple \\
\hline No & -0.334 & -0.068 & $0.002^{* * *}$ & $0.203^{* * *}$ & 3.62 & $26.54^{* * *}$ & $31.07^{* * *}$ & $31.96^{* * *}$ & Volatility Driven \\
\hline
\end{tabular}

Note: The above table presents the parameter estimates indicating the effects of US policy announcements on 58 digital assets. The sample is sorted into three cohorts, Currency, Protocol and dApp, based on the descriptions in Section 3. Coefficients $b^{2}, b^{4}$ and $b^{5}$ represent additional global systematic risk exposure during the announcement period (systematic volatility spillovers), additional US shocks during announcement period (idiosyncratic volatility spillovers) an intercept shift (shift volatility spillovers), respectively. $\pi^{2}$ represents additional US volatility spillovers during the announcement period (volatility driven spillovers). ${ }^{* * *}, * *$, and $*$ indicate level of significance at $1 \%, 5 \%$, and $10 \%$ respectively. 
Table 7: Cryptocurrency volatility spillovers based on two phases: QE (2013-2014) and Interest Rates (2015-2016)

\begin{tabular}{|c|c|c|c|c|c|c|c|c|}
\hline Asset & $b_{4}$ & $b_{5}$ & $b_{6}$ & $b_{7}$ & $b_{8}$ & $\pi_{1}$ & $\pi_{2}$ & $\pi_{3}$ \\
\hline AGRS & $0.426^{*}$ & - & -0.424 & - & 0.031 & $-0.014 * * *$ & - & $-0.021 * * *$ \\
\hline ANS & $1.020 * * *$ & - & $-0.895 * * *$ & - & $0.018^{* *}$ & $0.023^{*}$ & - & 0.003 \\
\hline BAY & $0.759^{* * *}$ & -0.143 & $-0.419^{*}$ & -0.009 & $0.004^{* * *}$ & $0.021 * * *$ & $-0.002^{* *}$ & $0.008^{*}$ \\
\hline $\mathrm{BCN}$ & $0.860^{* * *}$ & -0.205 & 0.282 & -0.001 & -0.005 & -0.028 & -0.004 & $0.006^{* * *}$ \\
\hline BLOCK & $0.827^{* * *}$ & -0.441 & 0.307 & 0.010 & 0.002 & -0.008 & $-0.014^{* * *}$ & 0.003 \\
\hline BTC1 & $0.151^{* *}$ & 0.632 & 0.322 & -0.003 & 0.007 & 0.062 & $-0.021^{*}$ & $0.015^{* *}$ \\
\hline BTCD & $0.830^{* * *}$ & 0.213 & $0.254^{* * *}$ & $0.023^{* * *}$ & $-0.067 * * *$ & $0.031^{*}$ & 0.014 & $-0.004^{* * *}$ \\
\hline BTS & $0.318^{* * *}$ & 0.191 * & $0.437^{*}$ & $-0.011 * *$ & 0.013 & $0.011 * * *$ & -0.008 & $0.014^{* * *}$ \\
\hline BURST & $0.907 * * *$ & 0.232 & -0.183 & $0.057 * * *$ & $0.032 * * *$ & $0.010 * * *$ & $0.009 * *$ & $-0.002^{* *}$ \\
\hline CLOAK & $0.712^{* * *}$ & -0.160 & -0.526 & 0.001 & -0.015 & $0.019^{* * *}$ & $-0.025^{* * *}$ & -0.002 \\
\hline DASH & $0.343^{* * *}$ & $0.536^{* * *}$ & $0.495^{* *}$ & 0.007 & 0.009 & $0.167^{* *}$ & 0.005 & 0.012 \\
\hline DCR & $0.707 * * *$ & 0.580 & -0.311 & $0.062^{*}$ & 0.020 & 0.040 & $0.008 * *$ & 0.005 \\
\hline DGB & $0.651^{* * *}$ & $0.524^{* * *}$ & $-0.445^{*}$ & $0.064 * * *$ & 0.009 & 0.027 & 0.007 & 0.016 \\
\hline DGD & $0.843^{* * *}$ & - & -0.205 & - & -0.010 & $0.013^{* * *}$ & - & $-0.011^{*}$ \\
\hline DOGE & $0.637^{* * *}$ & $0.232^{* * *}$ & $-0.232 * * *$ & 0.005 & -0.001 & $0.028^{* * *}$ & $0.011^{* * *}$ & $-0.003^{*}$ \\
\hline $\mathrm{EAC}$ & $0.614^{* * *}$ & $0.801 * * *$ & $-0.461^{* *}$ & $-0.014^{*}$ & $0.021^{* *}$ & $0.033^{* * *}$ & $-0.006^{* * *}$ & $0.005^{* * *}$ \\
\hline EMC & $0.782^{* * *}$ & -0.643 & -0.073 & 0.002 & 0.009 & $0.033^{* * *}$ & -0.006 & -0.003 \\
\hline ETC & $0.431 *$ & - & 0.196 & - & 0.001 & $0.062 * * *$ & - & $-0.003^{* *}$ \\
\hline ETH & $0.532^{* * *}$ & -0.464 & -0.096 & $0.028^{*}$ & 0.011 & $0.021^{* * *}$ & $0.003^{* * *}$ & -0.002 \\
\hline $\mathrm{FCT}$ & $0.364^{*}$ & -0.065 & $0.620^{*}$ & 0.002 & 0.003 & $0.014^{* * *}$ & $0.010^{* * *}$ & $-0.013^{* * *}$ \\
\hline GAME & $0.717^{* * *}$ & $-0.954^{* * *}$ & -0.086 & $0.033^{* *}$ & -0.004 & $0.017^{* *}$ & $0.014^{* * *}$ & $-0.002^{*}$ \\
\hline $\mathrm{ICN}$ & $0.401^{* * *}$ & - & 0.019 & - & 0.002 & $0.045^{* *}$ & - & $-0.003^{* * *}$ \\
\hline $\mathrm{LBC}$ & 0.529 & - & 0.201 & - & 0.005 & $0.052^{* * *}$ & - & $-0.005^{* *}$ \\
\hline LEO & $0.388^{* * *}$ & -0.397 & -0.215 & -0.010 & $-0.013^{* *}$ & $0.023^{* * *}$ & 0.009 & $-0.004^{* * *}$ \\
\hline LSK & $0.225 *$ & - & $0.313^{* *}$ & - & $0.014^{*}$ & $0.023^{* *}$ & - & -0.003 \\
\hline LTC & $0.964^{* * *}$ & -0.042 & $-0.323^{*}$ & -0.001 & 0.007 & $0.013^{* * *}$ & $0.005^{* * *}$ & $0.004^{* * *}$ \\
\hline MAID & $0.703^{* * *}$ & $0.448 * * *$ & $0.334^{*}$ & $0.029^{* * *}$ & 0.014 & $0.013^{* * *}$ & -0.002 & -0.004 \\
\hline MONA & $0.177^{* * *}$ & $0.444^{* * *}$ & $0.221^{*}$ & 0.003 & $0.013^{*}$ & $-0.011^{* *}$ & $0.004^{* * *}$ & $0.002^{* *}$ \\
\hline NLG & $0.792^{* * *}$ & 0.126 & -0.209 & 0.005 & 0.005 & $0.018^{* * *}$ & -0.016 & 0.006 \\
\hline NMC & $0.730^{* * *}$ & $0.221 * * *$ & 0.265 & $0.009 * * *$ & $0.014^{*}$ & $0.012^{* * *}$ & -0.004 & 0.015 \\
\hline NXS & $0.118^{* * *}$ & -0.785 & -0.704 & -0.011 & 0.001 & $0.090 * * *$ & 0.008 & $-0.013^{*}$ \\
\hline NXT & $0.843^{* * *}$ & 0.123 & $-0.133^{*}$ & $-0.029^{* * *}$ & 0.001 & $0.004^{*}$ & $0.005^{* * *}$ & -0.002 \\
\hline PIVX & $0.923^{* * *}$ & -0.122 & $-0.132 * * *$ & 0.074 & $0.047^{* *}$ & $0.024 * * *$ & $0.011 * * *$ & $-0.004 * * *$ \\
\hline PPC & $0.913^{* * *}$ & 0.082 & -0.154 & 0.004 & 0.007 & $0.012^{* * *}$ & $0.005^{* *}$ & $0.005^{*}$ \\
\hline RDD & $0.678^{* * *}$ & 0.365 & -0.223 & -0.002 & 0.004 & $0.028^{* * *}$ & $-0.008^{* * *}$ & $0.007 * * *$ \\
\hline REP & $0.668^{* * *}$ & - & 0.017 & - & 0.017 & $0.028^{* * *}$ & - & $-0.003^{* * *}$ \\
\hline $\mathrm{SC}$ & $0.105^{* * *}$ & -0.125 & 0.152 & $0.088^{* * *}$ & $0.017^{*}$ & $0.017^{* * *}$ & $-0.005^{* * *}$ & $0.007^{*}$ \\
\hline SJCX & $0.764^{* * *}$ & $-0.273^{*}$ & $-0.371^{*}$ & $0.075^{* * *}$ & 0.011 & $0.038^{* * *}$ & $-0.021 * * *$ & $-0.004 * * *$ \\
\hline $\mathrm{ST}$ & $0.495^{* *}$ & - & $-0.101 * * *$ & - & -0.008 & -0.011 & - & 0.004 \\
\hline STEEM & $-0.124 * * *$ & - & $0.187^{* * *} *$ & - & $0.023^{*}$ & $0.098 * *$ & - & $0.027 * * *$ \\
\hline SYS & $0.917^{* * *}$ & 0.069 & -0.185 & $0.015^{*}$ & 0.008 & 0.031 & 0.012 & 0.004 \\
\hline VIA & $0.785^{* * *}$ & 0.187 & -0.379 & 0.007 & $0.028^{* *}$ & $-0.035^{* * *}$ & $0.023^{* *}$ & $0.003^{*}$ \\
\hline VTC & $0.772^{* * *}$ & 0.179 & 0.544 & $-0.032^{* * *}$ & $0.083^{* * *}$ & 0.096 & 0.010 & 0.023 \\
\hline WAVES & $1.444^{* * *}$ & - & $-0.681 * *$ & - & 0.001 & $0.028^{* * *}$ & - & $-0.012^{* *}$ \\
\hline XAUR & $0.531 *$ & 0.652 & 0.578 & $-0.010^{*}$ & -0.016 & -0.007 & $-0.015^{*}$ & 0.127 \\
\hline $\mathrm{XCP}$ & $0.713^{* * *}$ & 0.095 & 0.277 & -0.022 & 0.005 & $-0.149^{*}$ & $0.008 * * *$ & $0.015 * * *$ \\
\hline XEM & $0.986^{* * *}$ & $0.245 * * *$ & -0.181 & $-0.040^{* *}$ & $0.016^{*}$ & $0.035 * * *$ & $0.024 * *$ & $0.004^{*}$ \\
\hline XLM & $0.484^{* * *}$ & -0.089 & 0.001 & 0.005 & 0.008 & 0.003 & -0.004 & 0.007 \\
\hline XMR & $0.941^{* * *}$ & 0.082 & -0.303 & 0.005 & 0.006 & $0.16^{* * *}$ & -0.004 & -0.006 \\
\hline XRP & $0.440^{* * *}$ & 0.042 & -0.115 & $0.008^{*}$ & -0.001 & $0.011^{* * *}$ & -0.004 & $-0.003 * * *$ \\
\hline XVG & $0.204^{* * *}$ & -0.115 & $-0.153^{*}$ & 0.032 & 0.018 & $0.028^{* * *}$ & $-0.003^{* * *}$ & $-0.004 * * *$ \\
\hline YBC & $0.243^{* * *}$ & $0.456^{* * *}$ & $0.198^{* * *}$ & $0.025^{* * *}$ & $-0.007 * * *$ & $0.018^{* * *}$ & 0.005 & 0.003 \\
\hline ZEC & $0.824^{*}$ & - & -0.076 & - & -0.004 & $0.006 * * *$ & - & $-0.005^{* * *}$ \\
\hline
\end{tabular}

Note: The above table presents the parameter results indicating the effects of US Policy announcements on 58 digital assets, based on 2 separate phases. For brevity, we have not presented the results of methodologies that did not present significant results. These results are available on request. The coefficients $b_{2}$ and $b_{3}$ represent additional global systematic risk exposure during each respective phase of announcement (systematic volatility spillovers). $b_{4}$ represents additional US shocks over the entire sample period. $b_{5}$ and $b_{6}$ represent additional US shocks during each respective phase (idiosyncratic volatility spillovers). $b_{7}$ and $b_{8}$ represent intercept shift (shift volatility spillovers) during each respective phase. $\pi_{1}$ represents US volatility spillover, during the entire sample period. $\pi_{2}$ and $\pi_{3}$ represent the additional US volatility driven spillovers that occurred during each respective announcement phase. ${ }^{* *}, * *$, and $*$ indicate level of significance at $1 \%$, $5 \%$, and $10 \%$ respectively. 